\title{
The Importance of Rapid Consideration of Creutzfeldt- Jakob Disease in the Differential Diagnosis of Progressive Neurodegenerative Disease: A Case Report
}

Arthur Joseph,' Jacob Core, ${ }^{1}$ Daniel Solano, ${ }^{1}$ Marquand Patton, ${ }^{2}$ Shaun Smart. ${ }^{2}$

\begin{abstract}
Background: Creutzfeldt-Jakob disease (CJD) is a prion disease characterized by misfolded proteins that lead to neurodegeneration and inevitable death. Classic sporadic CJD presents primarily with cognitive symptoms and ataxia without visual impairment at the onset of the illness. Seizure activity is a rare presentation of patients with sporadic CJD. Case: We present a rare case of rapidly progressive encephalopathy in a 57-year-old female who presented to the emergency department with bizarre behavior and vision deterioration. Imaging was unrevealing, and infectious and organic causes were ruled out. Electroencephalogram showed evidence of encephalopathy and non-convulsive status epilepticus. Magnetic resonance imaging conducted later displayed high signal intensity in centrum ovale. The patient's history, results from diagnostic analyses, and clinical presentation suggested the diagnosis of CJD (sporadic type). Conclusion: Due to the low incidence and varying clinical presentations, it is difficult to include CJD in a differential diagnosis without specific analytic measures. However, for the benefit of the patient and healthcare resources, CJD needs to be quickly considered when rapid neurological decline or non-convulsive status epilepticus is not suggestive of another entity.
\end{abstract}

Keywords: Creutzfeldt-Jakob Syndrome; Status Epilepticus; Diagnosis (Source: MeSH, NLM).

About the Author: Arthur Joseph is a student at Nova Southeastern University College of Osteopathic Medicine.
Submission: Oct 13, 2015 Acceptance: May 01, 2016 Publication: Aug 14, 2016 Process: Peer-reviewed

\section{Introduction}

Creutzfeldt-Jakob disease (CJD), the most iconic of the human prion pathologies, is characterized by misfolded proteins that lead to neurodegeneration and inevitable death. CJD displays an exceedingly fast rate of deterioration, with a median time from onset of symptoms to death of 12 months. The time from onset of symptoms to diagnosis varies, with a median of approximately 6 months. ${ }^{1}$ With an estimated incidence of one to two per million people annually, CJD is a rare but important consideration in the differential diagnosis of rapid neurodegeneration. ${ }^{2,3}$

Four major types of CJD exist: iatrogenic, variant, familial, and sporadic CJD. ${ }^{2}$ Sporadic CJD (sCJD) is responsible for $85 \%$ of CJD. ${ }^{3,4}$ Compared to other CJDS, SCJD has been shown to occur at a later age and affect women more frequently than men. ${ }^{5}$ Symptoms of SCJD include personality changes, sleep disturbances, cognitive function decline, behavioral abnormalities, visual abnormalities, hallucinations, cerebellar dysfunction, memory decline, myoclonus, progressive dementia, a positive startle reflex, and pyramidal dysfunction.

Several subtypes of SCJD have been studied. Classic SCJD has been described primarily with cognitive symptoms and ataxia without visual impairment at the onset of the illness. Classic SCID notoriously has a short interval between symptom onset and diagnostic evaluation. ${ }^{3}$ Seizure activity is a rare presentation in SCJD, and a review of the literature up to 2010 found that 12 patients have been reported to present with CID and

Key Points:
- Creutzfeldt-Jakob disease (CJD), particularly the sporadic subtype, is
a devastating disease with a rapid neurological degeneration pattern.
- In the case of unknown causes of neurodegenerative decline, CJD
should be included in the differential, and the laboratory testing should
be initiated early in management.
- A short window of time exists between symptom onset and death,
and diagnosis early in this window allows the patient to carry out his/
her final wishes and the family time for closure.

nonconvulsive status epilepticus (NCSE). ${ }^{6}$ In this report, we present a rare case of rapidly progressive encephalopathy in a 57-year-old female, who presented with cognitive decline and NCSE, which lead to the diagnosis of SCJD. All appropriate consents, including the use of this case for educational or research purposes, were obtained from the patient prior to admission.

\section{The Case}

A 57-year-old Hispanic female presented to our facility for an involuntary psychiatric evaluation for depression. The patient appeared disoriented and confused, with limited response to verbal/non-verbal cues and a flattened affect. According to the patient's spouse, the symptoms began three months prior to presentation with changes in vision and a decrease in responsiveness. Symptoms progressed, and the patient eventually had difficulty maintaining her employment. The spouse described her as having an unfocused gaze that fluctuated, difficulty with coordination, personality changes, and a decline in activities of daily living. She spent most of her day in silence. The day

Nova Southeastern University College of Osteopathic Medicine, Florida, USA.

Palmetto General Hospital, Florida, USA.

Correspondence:

Daniel Solano.

Address: Nova Southeastern University College of Osteopathic Medicine, 3301 College Ave, Fort Lauderdale, FL 33314, USA

Email: dsolano088@gmail.com 
after her admission, neurology specialist was consulted for the patient's altered mental status, and a full physical exam was limited by the patient's declining health. The initial computed tomography (CT) scan and magnetic resonance imaging (MRI) scan of her brain were unremarkable. An electroencephalogram (EEG) was also ordered to rule out an epileptic cause for the patient's episodes of unresponsiveness. Before the tests were administered, the patient was reported to have uncontrolled arm movements that prompted a transfer to the Emergency Room at Palmetto General Hospital. In the emergency room, tests for rapid plasma reagin (RPR), B12, folate, thyroid stimulating hormone (TSH), and ammonia levels were ordered. At this point, the patient was still mildly alert and able to follow simple commands. She did, however, exhibit bizarre behavior, including anger, anxiety and irritability.

The video EEG was very concerning, as it showed symmetric/ reactive background slowing activity at $5 \mathrm{~Hz}$ with intermittent triphasic morphology consistent with moderate-to-severe encephalopathy, as well as NCSE. Subsequent EEGs were consistent with these findings, and treatment was attempted with phenytoin (Dilantin). After anticonvulsant therapy failed to reverse or slow the rate of her neurodegeneration, a lumbar puncture was ordered for the analysis of $14-3-3$ and tau protein for a suspected rapidly progressing encephalopathy. After a week of deteriorating and inconclusive laboratory analysis, the patient's history of gastric band surgery prompted further laboratory tests. Her thiamine level was below normal limits, which prompted concern for the possibility of Wernicke or Korsakoff encephalopathy. Thiamine levels were replaced without an improvement in neurological function. Repeat EEGs continued to show signs of encephalopathy with generalized sharp wave discharges that appeared to be epileptiform in nature (Figure 1). While awaiting the results of $14-3-3$ and tau protein immunoassay, other laboratory tests returned negative results, including the tests for human immunodeficiency virus (HIV) and herpes simplex virus (HSV). At this point, the differential diagnosis was almost completely narrowed down to CJD. Autoimmune encephalitis, mitochondrial disease, heavy metal toxicity, paraneoplastic panel, derangements in amino acids, and organic acids and carnitine abnormalities were all ruled out.

Several days later, a repeat MRI revealed a single area of hyperintensity involving the right centrum semiovale consistent with a non-specific white matter lesion (Figure 2). The cerebrospinal fluid (CSF) was positive for $14-3-3$, and tau protein immunoassay revealed a tau protein level of $6,350 \mathrm{mg} / \mathrm{dL}$. The patient's clinical presentation, along with supporting results from MRI, EEG, 14-3-3 and tau protein immunoassay, all supported the diagnosis of CJD. While the definitive diagnosis was only achieved post-mortem, the findings were discussed with an expert in prion disease at the University of Chicago, who agreed with the likely diagnosis of CJD. The family, once informed of the findings, gave consent to genetic and post-mortem analysis. The patient later received hospice care, and the National Prion Surveillance Center had been informed of impending autopsy for genetic analysis.

\section{Discussion}

The patient presented with severe neurological dysfunctions as demonstrated by her disorientation, confusion, flat affect,
Figure 1. Repeat Electroencephalogram of the Patient

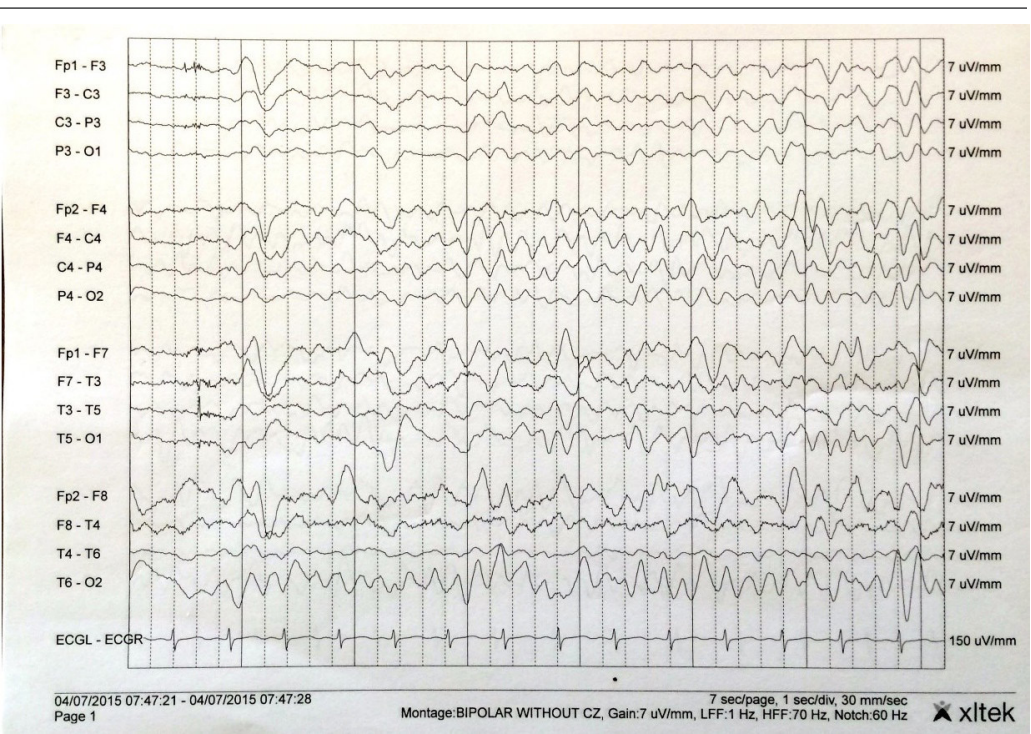

Electroencephalogram (EEG) of the patient displaying frequent triphasic spike and wave complexes. Discharges were frequent and at times continuous, lasting for hours. EEG was reviewed in a double banana montage with sensitivity at 7 microvolts and a time base of $30 \mathrm{~mm} / \mathrm{s}$. Background activity was seen primarily at $4-5 \mathrm{~Hz}$.

\section{Figure 2. Brain Magnetic Resonance Imaging (MRI) of the Patient}

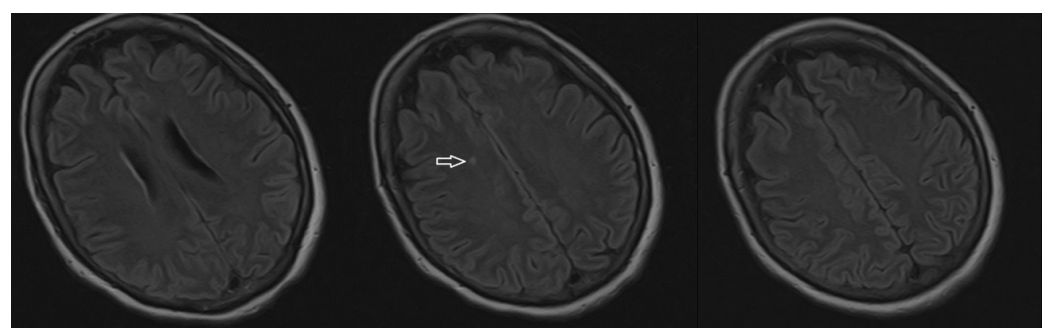

Three fluid-attenuated inversion recovery (FLAIR) magnetic resonance imaging (MRI) axial slices of the brain without gadolinium. Arrow: Single area of localized hyperintensity involving the right centrum semiovale above the lateral ventricles.

and limited responsiveness. However, retrospective questioning revealed that while these symptoms brought her to the hospital, other symptoms had been present for months. This considerable length of time is significant in that it represents a large portion of the total duration from disease onset to eventual death. Furthermore, CJD was only considered after the EEC demonstrated refractory NCSE and reversible causes of CJD had been ruled out, adding to the delay in probable diagnosis.

NCSE is an atypical finding in cases of SCJD, with only 12 cases being reported prior to this. ${ }^{6}$ Patients displaying this symptomatology were treated as having refractory NCSE and were non-responsive to multiple doses of antiepileptic drugs. Our patient was also unresponsive to several doses of phenytoin (Dilantin), which prompted the workup for sCJD.

The early stages of SCJD are largely nonspecific, making it difficult to suspect early on. As described earlier, subtle early symptoms include personality changes, sleep disturbances, and visual impairment. The classic finding of myoclonic jerks is typically seen later in the disease course and may be the eventual trigger for the SCJD workup. ${ }^{5}$ 
The diagnostic criteria for CJD outlined by the World Health Organization and Centers for Disease Control and Prevention (CDC) classify CJD into either definite, probable, or possible based on symptomatology, EEC findings, CSF analysis, and MRI (Table 1). Definitive diagnosis of CJD requires a tissue biopsy and is confirmed post mortem. However, updated criteria which may be superior to the current standard have been proposed.?

Diagnostic evaluation of patients with suspected SCJD involves assessment of the 14-3-3 protein assay, MRI, and EEG. Studies have demonstrated that CSF $14-3-3$ may be the most accurate test, with a sensitivity of $92 \%$ and a specificity of $80 \% .{ }^{8}$ Typical EEC findings of SCJD show periodic sharp and slow wave complexes (PSWC) with a reported sensitivity and specificity of $66 \%$ and $74 \%$, respectively. ${ }^{8}$ Our patient demonstrated these changes; however, wave complexes consistent with NCSE were also present. SCJD mimicking NCSE on EEG is extremely rare, as only 12 prior cases of CJD masquerading as NCSE have been documented. ${ }^{6}$ The importance of such finding has yet to be determined. MRI has shown to be the superior test by demonstrating areas of hyperintensity in the cortical and/or subcortical areas with sensitivity and specificity of $92.3 \%$ and $95 \%$, respectively. 8.9 Multiple studies found MRI to be superior in the evaluation of patients with suspected SCJD compared to EEG and 14-3-3 protein. ${ }^{9}$ With the demonstrated sensitivity and specificity of diffusion-weighted imaging (DWI) and fluid-attenuated inversion recovery (FLAIR) for white matter disease, MRI has become essential in the evaluation of rapidly progressive dementia. ${ }^{9}$

The National Prion Disease Pathology Surveillance Center (NPDPSC) provides CSF analysis for tau protein levels and the existence of 14-3-3 protein through real-time quaking-induced conversion (RT-QuiC) for the detection of abnormal prion protein. The center has reported a $98.5 \%$ specificity and up to $92 \%$ sensitivity in CSF analysis of 193 cases that have been autopsy-verified by the NPDPSC (Available from: http://case.edu/med/pathology/centers/npdpsc/index.html; cited 2015 May 31).

The RT-QuiC is reported as positive or negative. As reported by the NPDPSC, tau protein levels of $0-899 \mathrm{pg} / \mathrm{mL}$ correspond with a $25 \%$ probability of prion disease, $900-1,149 \mathrm{pg} / \mathrm{mL}$ with a $36 \%$ probability, and greater than $1,150 \mathrm{pg} / \mathrm{mL}$ with a $76 \%$ probability (Available from: http://case.edu/med/pathology/centers/ npdpsc/index.html; cited 2015 May 31). Our patient was positive for RT-QuiC and $14-3-3$ protein and had a t-tau protein level of $6,350 \mathrm{pg} / \mathrm{mL}$. After careful review of the literature, the consensus appears to be in agreement with the data reported by the NPDPSC. In fact, one study of 100 patients with varying neurodegenerative diseases found that significantly elevated levels of $\mathrm{t}$-tau protein was found in CJD alone (CJD and variant CJD) (Available from: http://www.who.int/zoonoses/diseases/ Creutzfeldt.pdf; cited 2015 Jun 25).

\section{Conclusion}

Ruling out reversible encephalopathic disease is important and potentially life-saving, but can be challenging and time consuming. CJD, particularly the sporadic subtype, is a devastating disease with a rapid neurological degeneration pattern. Due to the low incidence and varying clinical presentations, it is difficult to include CJD as a differential diagnosis without specific
Table 1. Diagnostic Criteria for Sporadic Creutzfeldt-Jakob Disease (sCJD).

\begin{tabular}{|c|c|}
\hline Possible Case & $\begin{array}{l}\text { - Progressive dementia; and } \\
\text { - Atypical EEG; and } \\
\text { - «2 year duration; and } \\
\text { - At least two of the following: } \\
\text { o Myoclonus } \\
\text { o Visual or cerebellar disturbance } \\
\text { o Pyramidal or extra pyramidal dysfunction } \\
\text { o Akinetic mutism }\end{array}$ \\
\hline $\begin{array}{l}\text { Probable Case (in } \\
\text { the absence of alter- } \\
\text { native diagnosis) }\end{array}$ & $\begin{array}{l}\text { - Progressive dementia; } \\
\text { - Two of the four clinical features mentioned } \\
\text { above for possible case, with } \\
\text { o A typical EEC of generalized triphasic } \\
\text { periodic complexes at the rate of one per } \\
\text { second; and/or } \\
\text { o Positive } 14-3-3 \text { assay in CSF; and } \\
\text { o MRI showing high signal abnormalities in } \\
\text { the caudate nucleus and/or putamen on } \\
\text { diffusion-weighted imaging (DWI) or } \\
\text { fluid-attenuated inversion recovery (FLAIR). } \\
\text { o Akinetic mutism }\end{array}$ \\
\hline Definite Case & $\begin{array}{l}\text { - Neuropathological confirmation post-mortem; } \\
\text { and/or } \\
\text { - Presence of protease-resistant prion protein } \\
\text { by either Western Blot or immunochemistry; } \\
\text { and/or } \\
\text { - Presence of scrapie associated fibrils. }\end{array}$ \\
\hline
\end{tabular}

and supportive analytic measures. In the case of unknown causes of neurodegenerative decline, it is imperative that CJD be included in the differential, and the laboratory testing should be initiated early in management. There exists a short window of time from symptom onset to death, and diagnosis early in this window allows the patient to carry out his/her final wishes and the family time for closure.

Unfortunately, in our patient, as well as the majority of patients who suffer from CJD, the diagnosis came late in the disease course, after significant neurological functions had deteriorated and the patient's quality of life had already been significantly impacted. Thus, CJD needs to be quickly considered when rapid neurological decline or NCSE is not suggestive of another entity.

\section{References}

1. González-Duarte A, Medina Z, Balaguer RR, Calleja JH. Can prion disease suspicion be supported earlier? Clinical, radiological and laboratory findings in a series of cases. Prion. 2011 Jul-Sep;5(3):201-7.

2. Head MW, Ironside JW. Review: Creutzfeldt-Jakob disease: prion protein type, disease phenotype and agent strain. Neuropathol Appl Neurobiol. 2012 Jun;38(4):296-310.

3. Appleby BS, Appleby KK, Crain BJ, Onyike CU, Wallin MT, Rabins PV. Characteristics of established and proposed sporadic Creutzfeldt-Jakob disease variants. Arch Neurol. 2009 Feb;66(2):208-15.

4. Prusiner SB. Shattuck lecture-neurodegenerative disease and prions. $\mathrm{N}$ Engl J Med. 2001 May 17;344(20):1516-26.

5. Zerr I, Poser S. Clinical diagnosis and differential diagnosis of CJD and VCJD. With special emphasis on laboratory tests. APMIS. 2002 Jan;110(1):88-98.

6. Espinosa PS, Bensalem-Owen MK, Fee DB. Sporadic Creutzfeldt-Jakob di- 
sease presenting as nonconvulsive status epilepticus case report and review of the literature. Clin Neurol Neurosurg. 2010 Jul;112(6):537-40.

7. Newey CR, Sarwal A, Wisco D, Alam S, Lederman RJ. Variability in diag nosing Creutzfeldt-Jakob disease using standard and proposed diagnostic criteria. J Neuroimaging. 2013 Jan;23(1):58-63.

8. Muayqil T, Gronseth C, Camicioli R. Evidence-based guideline: diagnostic accuracy of CSF 14-3-3 protein in sporadic Creutzfeldt-Jakob disease: report of the guideline development subcommittee of the American Academy of Neurology. Neurology. 2012 Oct 2;79(14):1499-506.

9. Bozluolcay M, Elmali AD, Menku SF, Zeydan B, Benbir G, Delil S, et al. Magnetic resonance imaging findings in probable Creutzfeld-Jacob disease: comparison with electroencephalography and cerebrospinal fluid characteristics. Acta Radiol Short Rep. 2014 Nov 14;3(10):2047981614552218.

\section{Acknowledgments}

None.

\section{Conflict of Interest Statement at Funding}

The authors have no funding, financial relationships, or conflicts of interest to disclose.

Author Contributions

Conceptualization, Data collection: AJ, JC. Data analysis and interpretation: AJ, JC, MP, SS. Writing: AJ, JC, DS. Critical revision of the manuscript: AJ, JC, DS, MP, SS. Approval of the final version: AJ, JC. Contribution of patients or study materials: MP, SS.

Cite as:

Joseph A, Core J, Solano D, Patton M, Smart S. The importance of rapid consideration of Creutzfeldt-Jakob disease in the differential diagnosis of progressive neurodegenerative disease: a case report. Int J Med Students. 2016 May-Aug;4(2):72-5. 